\author{
Pathophysiology \\ of Haemostasis \\ and Thrombosis
}

\title{
Self-Management of Oral Anticoagulants with a Whole Blood Prothrombin-Time Monitor in Elderly Patients with Atrial Fibrillation
}

\author{
Amiram Eldor ${ }^{\dagger}$ J oseph Schwart \\ Institute of Hematology, Tel-Aviv Sourasky Medical Center, Sackler Faculty of Medicine, Tel-Aviv University, \\ Tel-Aviv, Israel
}

\section{Key Words}

Atrial fibrillation - Oral anticoagulation .

Self-management . Point of care

\begin{abstract}
The efficacy of oral anticoagulants (OAC) in reducing the incidence of stroke in elderly patients with atrial fibrillation (AF) has been well documented. The intensity of OAC therapy and deviations in the prothrombin time (PT) are the strongest risk factor for bleeding complications in elderly patients. The aim of this study was to evaluate a more rigorous regulation of OAC by the use of a portable whole blood PT-monitor (CoaguChek) in elderly patients with AF (age 65-80 years). The study group consisted of 20 patients, of whom 17 were evaluable, which were trained to use to CoaguChek monitor and adjust their anticoagulant dose for 12 months. The control group, 20 patients matched for age, gender and the duration of OAC treatment, were tested in an anticoagulant clinic and their OAC dose was adjusted by a physician. To validate the PT-monitor results, the patients performed a total of 129 simultaneous venous blood PT tests at various time points. The correlation coefficient $R^{2}$ was 0.707 indicating the accuracy of the CoaguChek results. The self-managed patients perform more frequent measure-
\end{abstract}

ments $46 \pm 8.9$ vs. $15.7 \pm 3.1$ PT tests per patient. They demonstrated a within the therapeutic range INR in $80.5 \%$ of the tests (95\% confidence interval, $76.5-84.1 \%$ ) as compared to $72.4 \%$ (95\% confidence interval, $68.5-$ $76.5 \%)$ in the control group ( $p=0.057)$. The median value for all CoaguChek International Normalized Ratio (INR) recordings was within therapeutic range in the self-management group as well as in the control group. There were fewer INR results below or above the therapeutic range in the study group. None of the patients had hemorrhagic or thrombotic events during the study. Overall, the study group expressed high satisfaction from using the home monitor. We conclude that home PT monitoring and self-management of OAC are feasible in a motivated population of elderly patients with atrial fibrillation and are probably cost effective.

Copyright $\odot 2002$ S. Karger AG, Basel

\section{Introduction}

Atrial fibrillation (AF) is a common arrhythmia that is an important independent risk factor for stroke. The stroke rate in patients with $\mathrm{AF}$ is six times higher than without $\mathrm{AF}$ [1] and silent cerebral infarctions, which may impair cognitive functions, are frequently seen in asymp-

\begin{tabular}{ll}
\hline KARGER & ( 2002 S. Karger AG, Basel \\
1424-8832/02/0323-0099\$18.50/0 \\
$\begin{array}{l}\text { Fax +41613061234 } \\
\begin{array}{l}\text { E-Mail karger@karger.ch } \\
\text { www.karger.com }\end{array}\end{array}$ & $\begin{array}{l}\text { Accessible online at: } \\
\text { www.karger.com/journals/pht }\end{array}$
\end{tabular}

Joseph Schwartz, MD
Memorial Sloan Kettering Cancer Center
Box 531, 1275 York Avenue
New York, NY 10021 (USA)
Tel. +1 2126398603 , Fax +1 212717 3622, E-Mail eljosch@prodigy.net 
tomatic patients with $\mathrm{AF}[2,3]$. A recent meta-analysis [4] that included 9,874 patients showed that OAC significantly reduced the overall stroke rates for primary prevention as well as secondary prevention. Aspirin provided only a modest reductions, hence it was evident that warfarin is substantially more efficacious [4].

$\mathrm{AF}$ is a common condition among elderly patients. The prevalence of $\mathrm{AF}$ at the age of 60 years is $1 \%$, increasing to $5 \%$ for patients aged $70-75$ years and exceeding $10 \%$ in patients older than 80 years [5]. The rate of ischemic strokes in elderly patients with AF is rather high, $4.5 \%$ per year, and may be increased depending on the patient's age and the presence of valvular disease, prior thromboembolism, hypertension, diabetes, congestive heart failure and coronary heart disease [5]. Hence, AF has become the main indication for prolonged OAC therapy.

Older patients treated with OAC are more prone to bleeding, since they metabolize warfarin more slowly and have increased risk of drug interactions due to underlying chronic disease. Clinical trials have documented that the rate of major hemorrhages in patients treated with warfarin was $1.3 \%$ per year as compared to $1 \%$ in the placebotreated patients and central nervous system hemorrhages occurred in $0.3 \%$ of patients treated with warfarin compared to $0.1 \%$ in those treated with placebo $[5,6]$. However, the meta-analysis of the clinical trials showed that the benefit of OAC in AF patients was not offset by the occurrence of major hemorrhages [4]. A recent multicenter study has shown that deviation in the prothrombin time is the strongest risk factor for bleeding complications in elderly patients. An INR level above 4 substantially increases the risk of intracranial hemorrhage. Moreover, the intensity of OAC therapy was related to the incidence of life-threatening and fatal bleeding [4, 7]. Hence, it has been suggested that the best way to optimize OAC therapy and reduce the risk of intracerebral hemorrhages is to provide a better monitoring of the drug and target the INR values to a tight range of 2.0-3.0 [5, 8]. One of the options to provide more rigorous regulation of OAC is by the use of portable PT-monitors, which allow more convenient and thus more frequent control of OAC [9]. Point of care monitoring systems require a finger stick whole blood sample rather than citrated venous blood and the patient is capable of performing the assay at home. However, the use of such monitors for self-management of OAC has not been systematically evaluated in elderly patients with AF. We therefore initiated a prospective, feasibility study, in elderly patients (65-80 years) with paroxysmal or chronic $\mathrm{AF}$ to evaluate the use of the CoaguChek PT-monitor for the management of OAC. Self-management of OAC was compared to routine management by a physician in an anticoagulant clinic, in two groups of patients who were prospectively followed for one year.

\section{Patients and Methods}

\section{Study Population}

The study population consisted of elderly people (aged 65-80 years) with atrial fibrillation (mostly chronic), which received prophylactic OAC therapy with warfarin. The self-management group consisted of 20 patients who were instructed how to use the CoaguChek monitor and adjust their anticoagulant dose. These patients were accrued from an anticoagulant clinic and have been receiving $\mathrm{OAC}$ for at least 3 months prior to their enrollment. A control group of 20 patients was selected from the same anticoagulant clinic.

These patients were tested periodically as instructed and their anticoagulant dose was adjusted by a physician. The two groups were matched according to age, gender and duration of OAC treatment. Inclusion criteria to both groups were: patients who have used OAC for at least 3 months with reasonable monitoring results, signing an informed consent to participate in the study. Exclusion criteria were: disability, cognitive impairment, noncompliance, an abnormal blood count or coagulopathies. The study protocol has been approved by the hospital ethical committee and compiled with Helsinki II declaration.

\section{Whole Blood PT Monitor}

All self-managed patients used a capillary whole-blood prothrombin (PT) monitor (CoaguChek, Boehringer Mannheim, Germany). The monitor determines a PT from a finger stick sample of whole blood based on a thromboplastin induced clotting time. The test strip contains iron oxide particles, which are incorporated on the strip together with rabbit brain thromboplastin (international sensitivity index (ISI) value $=1.0$ ). This strip is inserted into the machine and a drop of blood $(10 \mu \mathrm{l})$ from a finger stick is applied to an application area and is carried by capillary action while mixing with the thromboplastin reagent at a temperature of $37^{\circ} \mathrm{C}$. The device contains two magnets, located beneath the test strip, which pulsate the iron oxide particles, and as soon as fibrin formation starts, the motility of the particles decreases. This change is recorded by reflectance photometry. A code chip with batch-specific calibration information is inserted, and the machine automatically converts the result into INR. The results of this monitor are not affected by hematocrit values between 32 and $55 \%$ or by fibrinogen concentrations between 100 and $500 \mathrm{mg} / \mathrm{dl}$, but they are affected by heparin. The claimed INR measuring range is $0.7-13.0$. The physical measures of the apparatus are $5.6 \times 14 \times 22 \mathrm{~cm}$ and the weight (including batteries) is $600 \mathrm{~g}$.

All the patients in the study group underwent a preparatory course of $5 \mathrm{~h}$. They received a lecture from a physician about the management of OAC and were instructed by a nurse how to perform a finger stick and how to use the CoaguChek monitor. The basic algorithm for adjusting the OAC dose was to use the same weekly dose of warfarin which the patients were used to from their previous experience in the anticoagulant clinic and to increase or reduce the weekly dose by $10 \%$, according to the changes in the INR. All the patients were asked to contact the investigators at any time of they had problems in dose adjustments. After 3 weeks, the patients met as a group 
and the study nurse checked their ability to perform the PT test. At the same time a routine laboratory PT test was performed and the results of both tests were compared and discussed with each patient individually. This procedure was repeated at 6 weeks.

\section{Study Protocol}

The duration of the study was 12 months. A therapeutic range of INR was considered between 1.9 and 3.1. The study group patients were supposed to perform PT tests once a week using the CoaguChek PT monitor. The patients were free to perform a PT measurement as often as they felt it was necessary. To validate the results, the patients were asked to come to the Hematology laboratory, where simultaneous capillary and venous blood PT testing was performed at 3 and 6 weeks after the initiation of the study and at 3, 6,9 and 12 months. The patients adjusted their warfarin dose by themselves according to the PT results. The control group patients performed their venous blood PT tests during their regular visits to the anticoagulant clinic at intervals determined by the physician in charge, and dose adjustments, if necessary, were made by the physician.

\section{Study End Points}

The study was designed to examine the following end points: the frequency of PT testing and the percentage of PT (INR) values within the therapeutic range in both groups. The accuracy of PT results was validated by the simultaneous testing using the fingerstick whole blood results and the routine PT results in citrated plasma. The attitude of the study patients to self-testing and self-management was determined by a questioner at the end of the study. The frequency of hemorrhagic and thromboembolic events was recorded for all the patients.

\section{Statistical Analysis}

All the INR values obtained from both groups were recorded using the Microsoft-Excel program. This allowed analysis of the data and statistical evaluation. Statistical significance level was set to 0.05 and SPSS software version 10 was used to perform the analysis and the charts. Differences between CoaguChek and laboratory INR values were displayed as a function of the laboratory INR value both for each individual patient and for the entire study group. Box and whiskers plots were made for both the self-management and control group in order to reveal median values and upper and lower quartiles. Outliners were marked with data points on the plots. The therapeutic efficacy of INR values within and outside the therapeutic range was compared by the Student's t test, and $95 \%$ confidence interval was calculated accordingly.

\section{Results}

\section{Patients and Controls}

Self-Managed Group. 38 patients were invited by the physician in charge of the anticoagulant clinic to participate in the self-management study group due to their skills and understanding of OAC management. However, only 20 patients agreed to sign the informed consent document. The reasons for not participating in the study were lack of interest (5 patients), fear of using the CoaguChek monitor and performing finger sticks ( 5 patients), and 8 patients did not want to leave the anticoagulation clinic due to social satisfaction. Of the patients who agreed to participate, all were high school graduates, 17 had an academic degree ( 3 physicians, 3 lawyers, $4 \mathrm{PhDs}$, one retired army general, 6 businessmen). One patient died of IHD during the study (death unrelated to OAC); 2 patients decided to leave the study, 3 and 4 weeks after initiation. 17 self-management patients completed the study period of 12 months and were considered in the overall data analysis. There were 15 males and 2 females. Mean age was 70.4 years (range 65-76).

Control Group. The control group consisted of 20 matched patient who were randomly selected by lottery from the OAC clinic. Three patients left the anticoagulant clinic during the study period (1, 2 and 4 months). 17 patients completed the study period of 12 months and were considered in the overall data analysis. There were 15 males and 2 females. Mean age was 73.4 (range 65-80) years.

\section{Clinical Course}

All the patients learned how to operate the PT monitor properly within 3 weeks and expressed confidence in the results. Two patients who had some initial problems had to receive additional instruction from the study nurse. There were only few phone calls to the investigators concerning dose adjustments and all the patients acquired confidence in adjusting their dose of OAC without further consultation. Two patients left the study within the first month. They felt reluctant to perform the assay and take responsibility for their OAC dose; they preferred to be followed in the anticoagulant clinic as before. The other patients expressed satisfaction to be independent from the anticoagulant clinic and stated that they preferred self-management over the management in the anticoagulant clinic. In fact, all 17 patients wanted to continue selfmanagement after the study period. 15 patients purchased the machine after completion of the study, while 2 patients had financial constraints.

There were no bleeding or thromboembolic complications in any of the patients during the study period.

\section{PT Testing}

The self-managed patients measured their PTs more frequently than the control group. A mean of $46( \pm 8.9)$ PT tests per patient were performed during a 12-month period by the study group as compared to $15.7( \pm 3.1)$ PT tests per patient in the control group. The self-management group demonstrated a within the therapeutic range 
Fig. 1. A box and whiskers plot of the median international normalized ratio (INR) value with one line in the box representing each patient. The corresponding upper/lower quartile (box) and 1.5 times the upper and lower quartiles (whiskers) are indicated as well. Outliners are marked as data points and asterisks on the plots. All the INR values were recorded.

\section{Self managed patients}

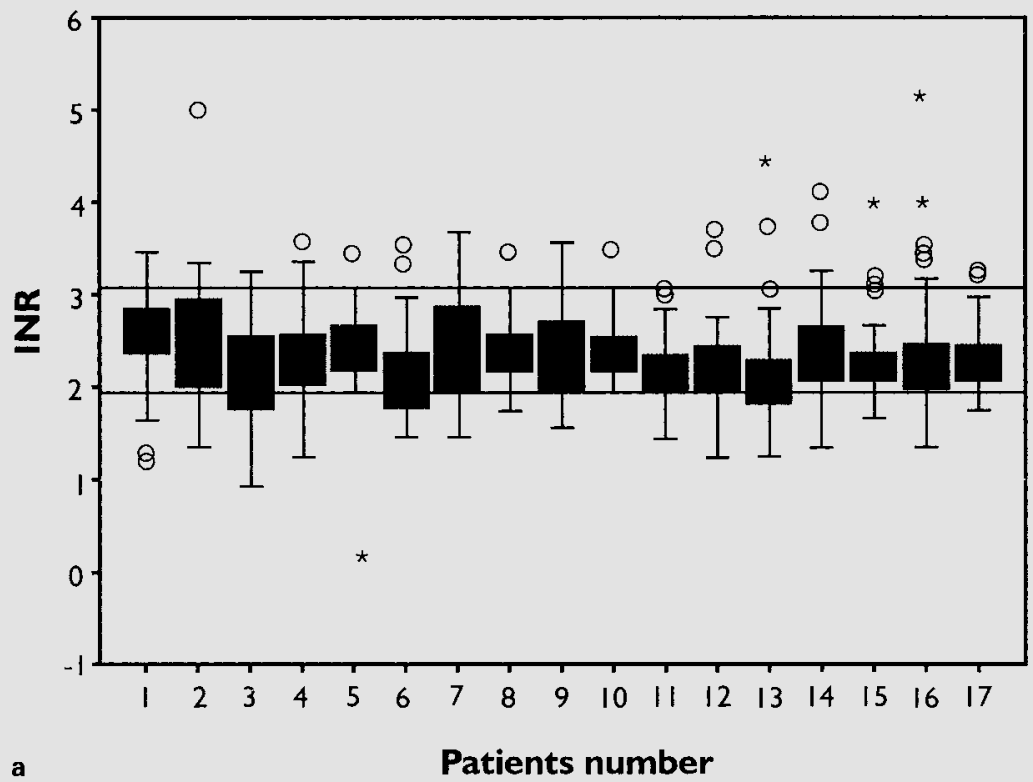

Control patients

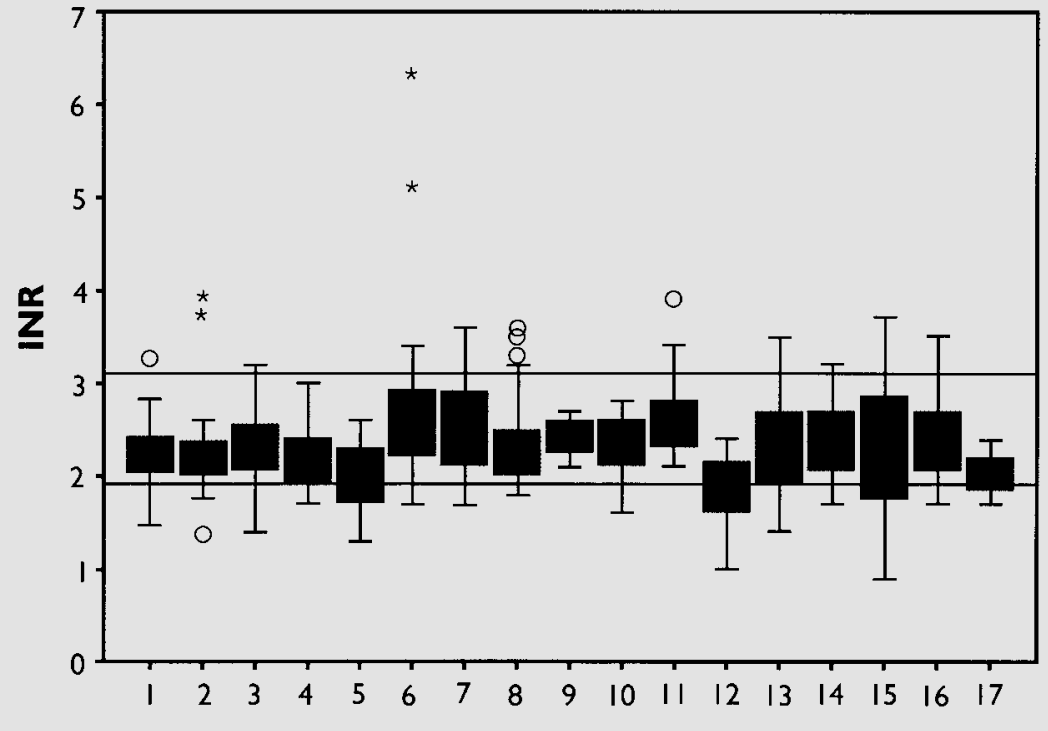

b
Patients number
INR in $80.5 \%$ (95\% confidence interval, $76.5-84.1 \%$ ) of the measurements, while the percentage test within the range in the control group was $72.4 \%$ (95\% confidence interval, 68.5-76.5\%) $(\mathrm{p}=0.057)($ table 1$)$. A higher percentage of patients in the control group had INR values below or above the therapeutic range compared to the study group (table 1). Figure 1a, b shows the box and whiskers plots for the self-management group and the control group. The median value for all CoaguChek INR recordings was within therapeutic range for all 17 patients 
Fig. 2. CoaguChek international normalized ratio (INR) values shown as a function of laboratory INR values for all the simultaneous measurements. The correlation coefficient $\left(R^{2}\right)$ is 0.707 .

Table 1. Laboratory results

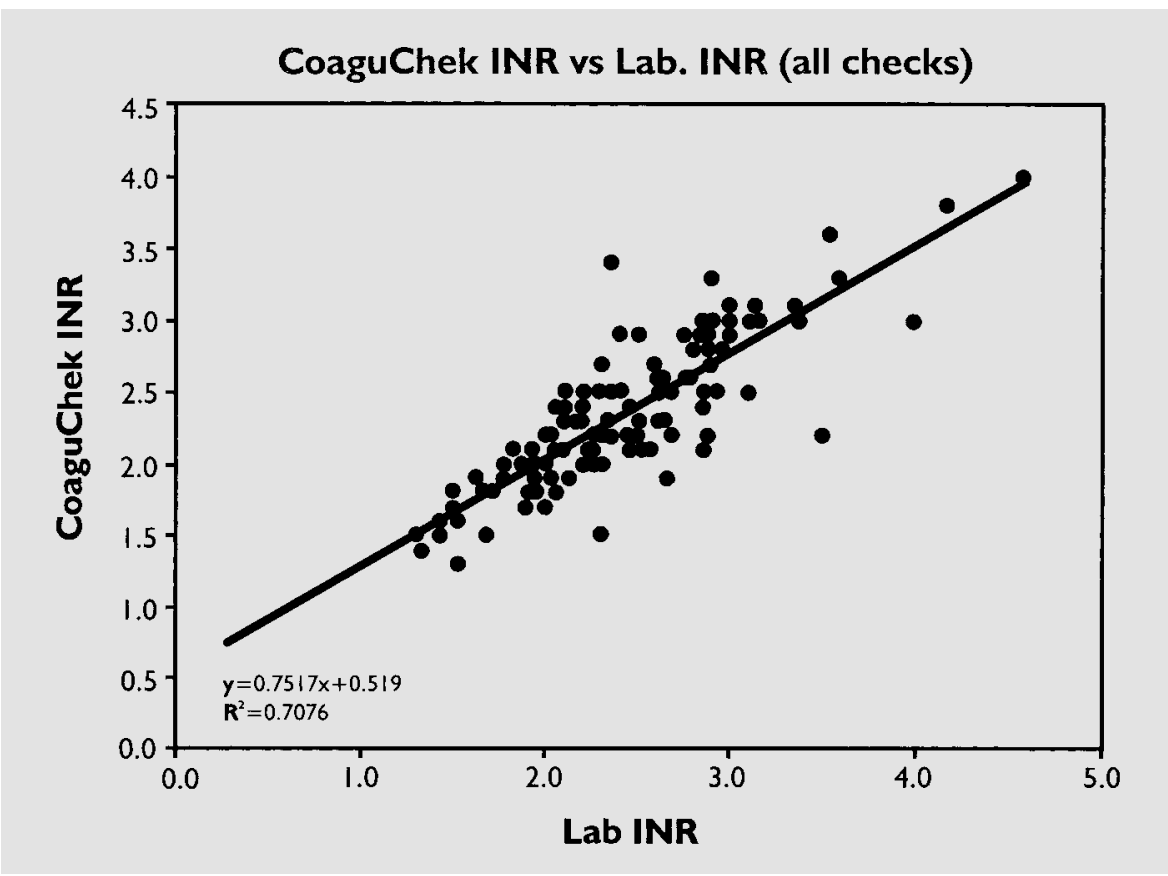

\begin{tabular}{|c|c|c|c|c|c|}
\hline \multirow[t]{2}{*}{ PT tests } & \multicolumn{2}{|c|}{ Self-managed patients } & \multicolumn{2}{|c|}{ Control patients } & \multirow[t]{2}{*}{$\mathrm{p}$} \\
\hline & $\mathrm{n}$ & $\%$ & $\mathrm{n}$ & $\%$ & \\
\hline Number of PT tests & 780 & & 268 & & \\
\hline PT tests/patient & 46 & & 15. & & \\
\hline INR results in range (1.9-3.1) & 628 & 80.5 & 194 & 72.4 & 0.057 \\
\hline INR results below range $(<1.9)$ & 92 & 11.8 & 48 & 17.9 & 0.13 \\
\hline INR results above range $(>3.1)$ & 60 & 7.7 & 26 & 9.7 & 0.52 \\
\hline INR 3.1-4 & 54 & 6.95 & 24 & 8.95 & \\
\hline INR 4-5 & 4 & 0.5 & 0 & & \\
\hline INR $>5$ & 2 & 0.25 & 2 & 0.75 & \\
\hline
\end{tabular}

in the self-management group as well as in the control group. Boxes and whiskers look similar for both groups, but the mean of range between upper and lower quartiles is 0.55 for the self-management group and 0.59 for the control group $(\mathrm{p}=0.06)$.

\section{Validation of the CoaguChek Monitor}

The study group patients performed at least six simultaneous PT tests using the CoaguChek monitor and routine a citrated venous blood PT, which was withdrawn within $5 \mathrm{~min}$ after the fingerstick and performed using the Sysmex apparatus, CA-6000, Sysmex, Japan. Some patients performed more simultaneous PT testing and alto- gether 129 tests performed by both methods were analyzed. Figure 2 shows the correlation between laboratory and the CoaguChek INR in the self-management group. $\mathrm{R}^{2}$ value, the correlation coefficient, was 0.7076 , which indicates significant agreement between both methods and high accuracy of CoaguChek INRs. The deviation form the ideal zero line of difference between the two analysis techniques is displayed for all 17 patients in figure 3. The median difference between all simultaneously drawn blood samples at the laboratory and CoaguChek monitor was $7 \%$ (0.14 INR value) or approximately 0.14 INR value at $I N R=2$.

Pathophysiol Haemost Thromb 2002;32:99-106 
Fig. 3. The difference between international normalized ratio (INR) values obtained in the laboratory and by the patients on the CoaguChek monitor are shown as a function of the laboratory INR for all the simultaneous measurements $(n=129)$ for all 17 study patients.

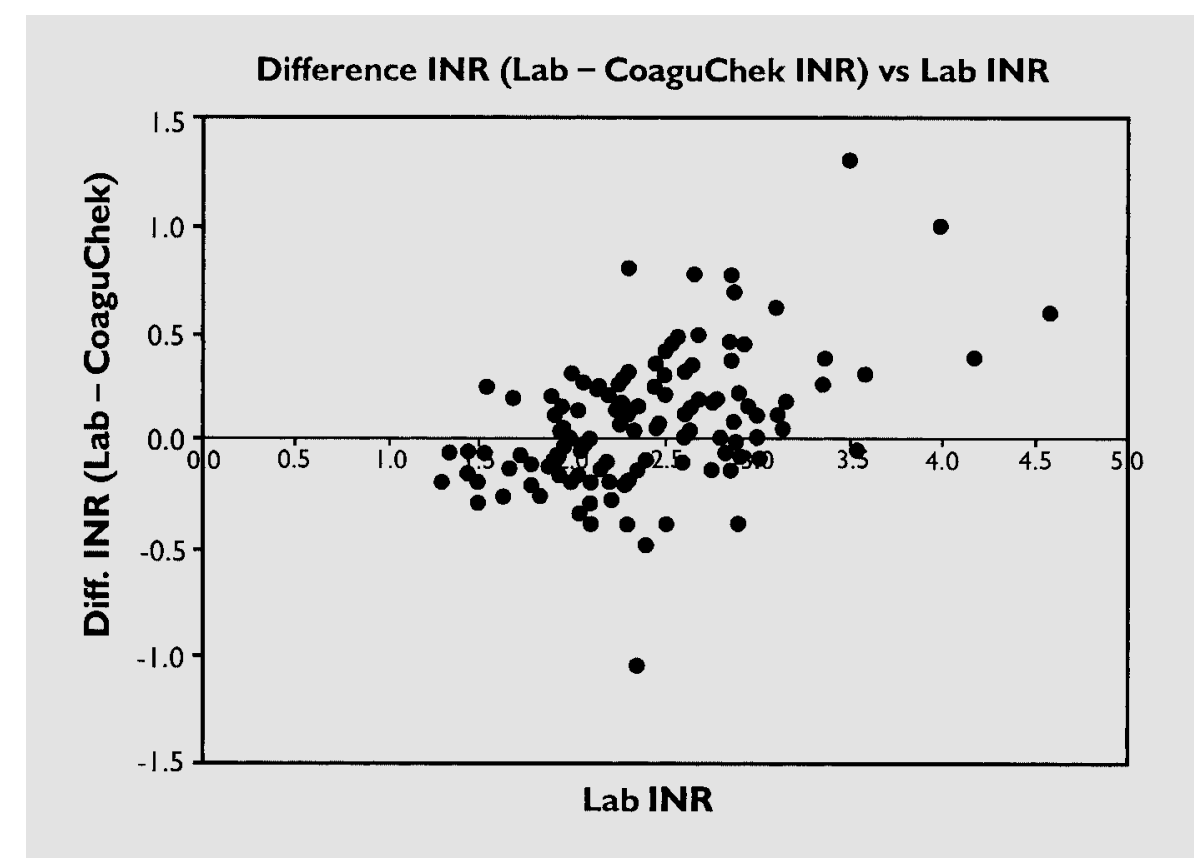

\section{Discussion}

Self-management of oral anticoagulation was first introduced in Germany in 1986. Since then several studies have shown that self-testing and self-management of OAC therapy might improve the quality of anticoagulation and reduce the incidences of bleeding complications and thromboembolic events both in adults and children [915]. It has become evident that the use of portable PT monitors has resulted in increased patient responsibility for monitoring their anticoagulation therapy. Currently, more than 40,000 patients in Germany determine the INR themselves and adjust their anticoagulant dose at home [9]. However, the majority of these are younger patients with prosthetic heart valves, which are highly motivated to perform the PT tests at home. No systematic studies have been performed in elderly patients with $\mathrm{AF}$, which is now the most frequent indication for prolonged OAC therapy.

The results of the present study demonstrated that selected elderly patients are capable of self-testing and self-managing of their OAC therapy. There were no significant differences in the percentage of INR values within the therapeutic range in both groups. The median value for all CoaguChek INR recordings was within therapeutic range in all 17 self-management group patients as well as in the control group, but the mean of range between upper and lower quartiles was smaller for the self-management group as compared to the control group. These data suggest that more frequent testing of the PT, as experienced by the self-management group, provides better results. The study indicated that self-testing and management of $\mathrm{OAC}$ is a very attractive feature for some patients who require life-long anticoagulation. It alleviated the need for frequent visits to the anticoagulant clinic, improved the compliance and allowed proper dosage adjustment of the OAC therapy. It alleviated fears of over- or underdosing that are common among anticoagulant users and increased the patient's self-confidence. Self-management gave the patients more responsibility for handling their disease and thus provided them with a better insight and motivation and better capacity to respond in real time to changes in their INR levels.

Despite the encouraging results, the study also suggested that many elderly patients with AF are not capable or interested of using the self-management system of OAC. Some have fears of using the monitor, some are afraid or are incapable of adjusting the OAC dose, and many patients, mostly the retired ones, were reluctant to giving up their monthly visits to the physician and their friends in the anticoagulant clinic. Hence, the use of the PT monitors should be suggested to elderly patients who conduct an active life, which indeed was the case in the group which agreed to participate in this study. A careful selection of the patients by the responsible physician is important for a successful self-management of OAC. The intel- 
lectual abilities should be sufficient to understand the concept of OAC therapy and its potential risks and the patients should show sufficient dexterity and demonstrate willingness to participate actively in their own treatment.

Another important aspect of using the portable PT monitors is to provide an appropriate educational program which will include proper handling and operating of the monitor and an adequate finger stick technique as well as theoretical knowledge of OAC therapy with major focus on the adjustment of the anticoagulant dose depending on the INR values measured. Structured teaching for self-management of OAC has recently been evaluated [16]. It is our opinion that teaching has to be tailored for different patient populations. In Germany, the patients receive a preparatory course of 2 days and undergo testing following which they receive the PT monitor and start to adjust their OAC doses. We thought that this method of structured teaching is not suitable for our elderly patients for many reasons. Hence, we decided to accept patients into the study only if they had attended the anticoagulant clinic for at least 3 months. We assumed that during this period, the patients acquired enough experience with the management of their OAC therapy and were capable of better understanding the adjustments needed for certain changes in the INR. We believe that the good results achieved in our patients were mainly due to their previous experience. Hence, we would suggest that every new patient who starts OAC therapy and is interested in acquiring a portable PT monitor would first be followed in the regular anticoagulant clinic for a period of time which will allow him and his physician to discuss various issues of dose adjustment and self-management.

Our study as well as other studies clearly demonstrated that the results obtained with the CoaguChek monitor were reliable and well correlated with the routine laboratory PT results [16-18]. Recently, Chapman et al. [19] compared the CoaguChek with the routine laboratory and found no significant differences between the two methods. Another multicenter study using the CoaguChek monitor demonstrated a mean within-run and day-to-day coefficient of variation of $7 \%$ and an overall coefficient of variation of approximately $8 \%$ [21]. These results confirm that PT testing from capillary blood samples is of sufficient precision to yield reliable INR results in patient selftesting.

The clinical outcome in terms of bleeding or thrombotic complications could not be properly evaluated since the number of patients in our study was small and the duration of the study was too short. Various studies have shown that patients who control their OAC therapy by self-testing perform the tests more frequently, as was the case in our study [11-14]. More frequent determinations of the INR decrease the proportion of INR values outside the range and thus may decrease the complications. Indeed, it was recently demonstrated in a retrospective study that more frequent self-testing which caused the patient to be within the INR target range for a longer period of time, reduced thromboembolic events and hemorrhagic complications [22].

Several monitors are currently available for fingerstick testing of the PT. These include CoaguChek and the CoaguChek Plus (manufactured by Roche Diagnostics, Germany) and ProTime Microcoagulation System (manufactured by International Technidyne Corporation, USA). Single tests performed with these monitors are more expensive than the price of a routine PT test. However, cost effectiveness should take into consideration a prolonged use of the monitor saving the costs of the monitor as well as visits to the anticoagulant clinic and the reduction in the hemorrhagic or thromboembolic events whit better anticoagulation. Studies from the USA suggested the cost-effectiveness of portable PT Monitors [23], and a recent German study showed that home monitoring of the INR decreased the costs of treatment by $35 \%$ per patient-year in comparison to conventional monitoring [24].

Atrial fibrillation is 'the epidemic of the millennium' [25]. Elderly patients who will develop AF will need a simple, cost-effective treatment strategy to prevent silent cerebral as well as the more disabling and sometimes fatal ischemic strokes. Warfarin is currently the drug of choice. It is inexpensive; however, its risk benefit ratio margins are rather narrow and frequent adjustments of the dose are necessary. Self-management of oral anticoagulation with a portable whole blood PT monitor is convenient for the patient, improves the quality of OAC therapy and may reduce the risk of bleeding and thromboembolic events. Until new antithrombotic drugs, which do not need careful monitoring, will become available, this new approach that is probably cost-effective, should be recommended to suitable, motivated elderly patients with atrial fibrillation.

\section{Acknowledgements}

The authors are thankful to Dr. Harald Wolf and Roche Diagnostics Co, Mannheim, Germany, for supporting this trial; to Mrs. Ronit Elon for assisting the patients and to Ms E. Eshkol for editorial assistance.

Pathophysiol Haemost Thromb 2002;32:99-106 


\section{References}

1 Laupacis A, Albers G, Dalen G, Dunn M, Feinberg W, Jacobson A: Antithrombotic therapy in atrial fibrillation. Chest 1995;108:352S$359 \mathrm{~S}$.

2 Ezekowitz MD, James KE, Nazarian SM, et al: Silent cerebral infarction in patients with nonrheumatic atrial fibrillation. Circulation 1995 ; 92:2178-2182.

3 Kilander L, Andren B, Ny H, Lind L, Boberg $\mathrm{M}$, Lithell $\mathrm{H}$ : Atrial fibrillation is an independent determinant of low cognitive function: A cross-sectional study in elderly men. Stroke 1998;29:1816-1820.

4 Hart RG, Benavente O, McBride R, Pearce LA: Antithrombotic therapy to prevent stroke in patients with atrial fibrillation. Ann Intern Med 1999;131:492-501.

5 Ezekowitz MD, Levine JA: Preventing stroke in patients with atrial fibrillation. JAMA 1999; 281:1830-1835.

6 Hart RG, Pearce LA, McBride R, Rothbart RM, Asinger RW: For the Stroke Prevention in Atrial Fibrillation (SPAF) Investigators: Factors associated with ischemic stroke during aspirin therapy in atrial fibrillation. Stroke 1999 30:1223-1229.

7 Hart RG: Intensity of anticoagulation to prevent stroke in patients with atrial fibrillation. Ann Intern Med 1998;128:408.

8 Hylek EM, Skates SJ, Sheehan MA, Singer DE: An analysis of the lowest effective intensity of prophylactic anticoagulation for patients with nonrheumatic atrial fibrillation. N Engl J Med 1996;335:540-546.

9 Taborski U, Muller-Berghaus G: State-of-theart patient self-management for control of oral anticoagulation. Semin Thromb Hemost 1999,25:43-48.
10 White RH, McCurdy SA, Von Marensdorff $\mathrm{H}$, Woodruff DE Jr, Leftgoff L: Home prothrombin time monitoring after the initiation of warfarin therapy: A randomized, prospective study. Ann Intern Med 1989;11:730-736.

11 Anderson Dr, Harrison L, Hirsh J: Evaluation of a portable prothrombin time monitor for home use by patients who require long-term oral anticoagulant therapy. Arch Intern Med 1993; 153:1441-1447.

12 Ansell JE, Patel N, Ostrovsky D, Nozzolillo E, Peterson AM, Fish L: Long-term patient selfmanagement of oral anticoagulation. Arch Intern Med 1995; 155:2185-2189.

13 Massicotte P, Marzinotto V, Vegh P, Adams M, Andrew M: Home monitoring of warfarin therapy in children with a whole blood prothrombin time monitor. J Pediatr 1995;127: 389-394.

14 Hasenkam JM, Kimose HH, Knudsen L, et al: Self management of oral anticoagulant therapy after heart valve replacement. Eur J Cardiothorac Surg 1997;11:935-942.

15 Bernardo A: Post-conference session: Experience with patient self-management of oral anticoagulation. J Thromb Thrombolysis 1996;2: 321-325.

16 Sawicki PT: A structured teaching and selfmanagement program for patients receiving oral anticoagulation: A randomized controlled trial. JAMA 1999;281:145-150.

17 Kaatz SS, White RH, Hill J, Mascha E, Humphries JE, Becker DM: Accuracy of laboratory and portable monitor international normalized ratio determinations. Arch Intern Med 1995; 155:1861-1867.
18 Douketis JD, Lane A, Milne J, Ginsberg JS: Accuracy of a portable international normalization ratio monitor in outpatients receiving long-term oral anticoagulant therapy: Comparison with a laboratory reference standard using clinically relevant criteria for agreement. Thromb Res 1998;92:11-17.

19 Vacas M, Lafuente PJ, Cuesta S, Iriartem JA Comparative study of a portable monitor for prothrombin time determination, CoaguChek, with three systems for control of oral anticoagulation treatment. Haemostasis 1998;28:321328.

20 Chapman DC, Stephens MA, Hamann GIL, Bailey LE, Dorko CS: Accuracy, clinical correlation and patient acceptance of two handheld prothrombin time monitoring devices in the ambulatory setting. Ann Pharmacother 1999; 33:775-780.

21 Van den Besselaar AM, Breddin K, Lutze G, et al: Multicenter evaluation of a new capillary blood prothrombin time monitoring system. Blood Coagul Fibrinol 1995;6:726-732.

22 Bussey HI, Chiquette E, Amato MG: Workshop: anticoagulation clinic care versus routine medical care: A review and interim report. J Thromb Thrombolysis 1996;2:325-329.

23 Ansell JE, Hughes R: Evolving models of warfarin management: Anticoagulation clinics, patient self-monitoring, and patient self-management. Am Heart J 1996;132:1095-1100.

24 Taborski U, Wittstamm FJ, Bernardo A: Costeffectiveness of anticoagulation self-management in Germany. Semin Thromb Hemostas 1999;25:103-109.

25 Ezekowitz MD: Atrial fibrillation: The epidemic of the millennium. Ann Intern Med 1999; 131:537-538. 